\title{
A new analysis procedure to extract fusion excitation function with large beam energy dispersions: application to the ${ }^{6} \mathrm{Li}+{ }^{120} \mathrm{Sn}$ and ${ }^{7} \mathrm{Li}+{ }^{119} \mathrm{Sn}$
}

\author{
Alessia Di Pietro ${ }^{1, \star}$, Pierpaolo Figuera ${ }^{1}$, Maria Fisichella ${ }^{1}$, Alan C. Shotter ${ }^{2}$, Juan Pablo Fernandez-Garcia ${ }^{1,3}$, Jonas \\ L. Ferreira ${ }^{4}$, Marcello Lattuada ${ }^{1,3}$, Paolo Lotti $^{5}$, Jesus Lubian ${ }^{4}$, Chris Ruiz ${ }^{6}$, Domenico Torresi ${ }^{1,3}$, and Mile Zadro ${ }^{7}$ \\ ${ }^{1}$ INFN, Laboratori Nazionali del Sud, via S. Sofia 62, I-95123 \\ ${ }^{2}$ School of Physics and Astronomy, University of Edinburgh, JCMB, Mayfield Road, Edinburgh EH9 3JZ, UK \\ ${ }^{3}$ Dipartimento di Fisica ed Astronomia, Universitá di Catania, via S. Sofia 64, I-95123 Catania, Italy \\ ${ }^{4}$ Instituto de Física, Universidade Federal Fluminense, Avenida Litoranea s/n, Gragoatá, Niterói, Rio de Janeiro 24210-340, Brazil \\ ${ }^{5}$ INFN, Sezione di Padova, Via F. Marzolo 8, 35131, Padova, Italy \\ ${ }^{6}$ TRIUMF 4004 Wesbrook Mall Vancouver BC V6T 2A3, Canada \\ ${ }^{7}$ Ruđer Bošković Institute, Bijenička cesta 54,HR-10000 Zagreb, Croatia
}

\begin{abstract}
.
In the present paper it is described an analysis procedure suited for experiments where cross-sections strongly varying with energy are measured using beams having large energy dispersion. These cross-sections are typically the sub-barrier fusion excitation function of reactions induced by radioactive beams. The large beam energy dispersion, typical of these experiments, can lead to ambiguities in the association of the effective beam energy to the reaction product yields and consequently to an error in the determination of the excitation function. As a test case, the approach is applied to the experiments ${ }^{6} \mathrm{Li}+{ }^{120} \mathrm{Sn}$ and ${ }^{7} \mathrm{Li}+{ }^{119} \mathrm{Sn}$ measured in the energy range $14 \mathrm{MeV} \leq \mathrm{E}_{\mathrm{c} . \mathrm{m} .} \leq 28 \mathrm{MeV}$. The complete fusion cross sections are deduced from activation measurements using the stacked target technique. The results of these experiments, that employ the two weakly-bound stable $\mathrm{Li}$ isotopes, show that the complete fusion cross sections above the barrier are suppressed of about $70 \%$ and $85 \%$ with respect to the Universal Fusion Function, used as a standard reference, in the ${ }^{6} \mathrm{Li}$ and ${ }^{7} \mathrm{Li}$ induced reactions respectively. Moreover, the excitation functions of the two systems at energies below the barrier, do not show significant differences, despite the two systems have different $n$-transfer $\mathrm{Q}_{\text {value }}$.
\end{abstract}

\section{Introduction}

The stacked target technique has been used in many experiments to measure excitation functions of reactions, such as for example fusion, induced by low energy radioactive beams e.g. [1-5]. In order to increase the production yields of the reaction products, thick targets have also been employed in some of these experiments. The advantage of using the stacked target technique consists in measuring the cross-section at several energies simultaneously; as a drawback, however, there is the degradation of the beam quality as it passes through the foils of the stack, due both, to the statistical nature of the energy loss process, and to any target non-uniformities. Ambiguities of associating effective beam energies to reaction product yields for the targets within the stack may arise and, as a consequence, an error in the extraction of the excitation function for the reaction under study. In [6] the possible sources of ambiguities arising when performing experiments with large beam energy spread were investigated thoroughly. Typically, the large beam energy dispersions are due either to the use the stacked target technique and/or thick-non-uniform targets, or to the beam production tech- nique. Moreover, in [6], a best practice procedure to extract cross-sections strongly varying with energy obtained with large beam energy dispersions was proposed. In the present paper the technique proposed in [6] will be summarized and compared to the iterative process suggested in [7]. In addition, the analysis procedure proposed in [6] is applied to the measurements of ${ }^{6} \mathrm{Li}+{ }^{120} \mathrm{Sn}$ and ${ }^{7} \mathrm{Li}+{ }^{119} \mathrm{Sn}$ fusion excitation functions, measured in the energy range from $14 \mathrm{MeV}$ to $28 \mathrm{MeV}$ in the center of mass. The aim of these experiments is twofold: to investigate the effect of neutron transfer channels with positive $\mathrm{Q}_{\text {value }}$ on the subbarrier fusion probability e.g. [8-12], and the suppression of the complete fusion cross-section above the barrier in reactions induced by weakly-bound beams, with respect to one-dimensional Barrier Penetration Model (1D-BPM), or coupled-channel (CC) calculations which do not take coupling to continuum or transfer into account (see e.g. [13-17]).

\footnotetext{
^e-mail: dipietro@1ns.infn.it
} 


\section{Experimental determination of the fusion excitation function}

The mean cross-section, $\sigma_{\text {mean }}$, of a given reaction process, is experimentally deduced by using the equation:

$$
\sigma_{\text {mean }}=\frac{Y}{N_{t_{0}} \cdot N_{B}}
$$

where $N_{t_{0}}$ is the number of atoms per unit area of the target, and $N_{B}$ the number of beam particles passing through the target. If one has to derive an excitation function, the problem is how to relate these measured mean cross-sections to effective beam energies. Generally it is assumed $\sigma_{\text {mean }}=$ $\sigma(\bar{E})$ where $\bar{E}$ is the energy in the centre of the target, as calculated by energy-loss programs. Alternatively, it has been assumed [5] that $\sigma_{\text {mean }}=\sigma\left(\mathrm{E}_{\text {eff }}\right)$, where $E_{\text {eff }}$ is the weighted mean energy defined by eq. 2 :

$$
E_{e f f}=\frac{\int_{0}^{\infty} E \sigma(E) D\left(E, t_{0}\right) d E}{\int_{0}^{\infty} \sigma(E) D\left(E, t_{0}\right) d E}
$$

The weighting factors are the cross-section $\sigma(\mathrm{E})$ and the beam energy probability distribution function $D\left(E, t_{0}\right)$ inside the target. $D\left(E, t_{0}\right)$ represents the probability to find a beam particle with energy $E$ inside a given target with average thickness $t_{0}$.

In the case of large beam energy dispersion, and when the cross section is known to vary rapidly over the energy range explored by the beam in the target, the above methods of relating $\sigma_{\text {mean }}$ to $\sigma(\mathrm{E})$ can result in misinterpretation of the real excitation function.

The measured mean cross sections $\sigma_{\text {mean }}$, are energy mean values of the real excitation function $\sigma(\mathrm{E})$ and, as discussed in [6], can be specified for a given measurement $i$ as:

$$
\sigma_{\text {mean }, i}=\frac{\int_{0}^{\infty} \sigma(E) D_{i}\left(E, t_{0}\right) d E}{\int_{0}^{\infty} D_{i}\left(E, t_{0}\right) d E},
$$

The problem of deducing the correct $\sigma(\mathrm{E})$ from the measured $\sigma_{\text {mean }}$ can be solved by choosing a suitable function $\hat{g}(E, \mu)$ (with $\mu$ parameters to be determined) which shows the same expected energy behavior as $\sigma(E)$. In [6] it was suggested an unfolding procedure to deduce the correct $\sigma(\mathrm{E})$. The mean value of the function $\hat{g}(E, \mu)$ over the energy range explored by the beam in the target is given by eq. 4 :

$$
\hat{g}_{\text {mean }, i}=\frac{\int_{0}^{\infty} \hat{g}(E, \mu) D_{i}\left(E, t_{0}\right) d E}{\int_{0}^{\infty} D_{i}\left(E, t_{0}\right) d E},
$$

The function $\hat{g}(E, \mu)$ can be obtained by minimizing the following expression with respect to $\mu$ :

$$
S=\sum_{i}\left(\frac{\sigma_{\text {mean }, i}-\hat{g}_{\text {mean }, i}}{\beta_{i}}\right)^{2}
$$

where $\beta_{i}$ is the experimental error associated with $\sigma_{\text {mean }, i}$.

In order to apply the above procedure, in addition to finding the proper $\hat{g}(E, \mu)$, it is required the precise knowledge of $D_{i}\left(E, t_{0}\right)$; this can be deduced, with a good degree of approximation, using the code SRIM [18, 19].

\section{Determination of the energy probability distribution for uniform and non uniform foils}

The beam energy probability distribution is given by eq. 6:

$$
D\left(E, t_{0}\right)=\int_{0}^{\infty} g\left(E_{0}, E_{i}\right) \int_{0}^{t_{0}} f\left(E_{i}, t_{0}, E, x^{\prime}\right) d x^{\prime} d E_{i}
$$

this, as mentioned above, represents the probability to have a beam particle with energy $E$ inside the considered target of thickness $t_{0} . f\left(E_{i}, t_{0}, E, x^{\prime}\right)$ represents the probability that a projectile incident on the target of thickness $t_{0}$ at an incident energy $E_{i}$ has an energy $E$ at a depth $x^{\prime}$ inside the target, with $0<x^{\prime}<t_{0} . g\left(E_{0}, E_{i}\right)$ represents the probability that a particle, as part of the entrance beam of mean energy $E_{0}$, has an energy $E_{i}$. In many cases, $g\left(E_{0}, E_{i}\right)$ is usually considered a Gaussian distribution, however, as shown in [6], this is not always the case.

The SRIM code can be used for determining $f\left(E_{i}, t, E, x\right)$ and $g\left(E_{0}, E_{i}\right)$ with a procedure discussed in detail in [6]. In general, targets are non-uniform, so to explicitly account for target non-uniformity Eq. (6) should include another term, the thickness probability distribution function, $w(t)$, which represents the probability that a particle entering a non-uniform target actually experiences a thickness $t$. In this case the energy distribution inside the target will be defined as follows:

$$
\begin{aligned}
& D\left(E, t_{0}\right)= \\
& \int_{0}^{t_{0}} w(t) \int_{0}^{\infty} g\left(E_{0}, E_{i}\right) \int_{0}^{t} f\left(E_{i}, t, E, x^{\prime}\right) d x^{\prime} d E_{i} d t
\end{aligned}
$$

with $0<x^{\prime}<\mathrm{t}$, and $t_{0}$ represents the maximum target thickness. Therefore, for determining $D_{i}\left(E, t_{0}\right)$ it is necessary to know $w(t)$. The probability distribution function $w(t)$ of a given target can be determined experimentally using the residual energy spectra of $\alpha$-particles emitted from a ${ }^{241} \mathrm{Am}$ source passing through the target, and SRIM simulations. Using SRIM, the residual energy spectra of the ${ }^{241} \mathrm{Am} \alpha$-particles, passing though a ${ }^{93} \mathrm{Nb}$ foil plus a ${ }^{120} \mathrm{Sn}$ foil whose thickness was varied from $0 \mathrm{mg} / \mathrm{cm}^{2}$ to $1.5 \mathrm{mg} / \mathrm{cm}^{2}$, were calculated. A weight, $w(t)$, was attributed to each of the individual calculated spectra such that their weighted sum was reproducing the experimental residual energy spectrum. Fig. 1 represents the experimental residual energy spectrum of $\alpha$-particles crossing a ${ }^{120} \mathrm{Sn}$ target evaporated onto a ${ }^{93} \mathrm{Nb}$ substrate, used in the ${ }^{6} \mathrm{Li}+{ }^{120} \mathrm{Sn}$ experiment, compared with the spectrum obtained with the SRIM simulations. In Fig. 2 the corresponding $w(t)$ is reported as function of the target thickness. In this specific case the ${ }^{120} \mathrm{Sn}$ targets used in the experiment were highly non-uniform. It seems that high non-uniformities are typical of Sn targets, unless special care is taken in the target production procedure to 


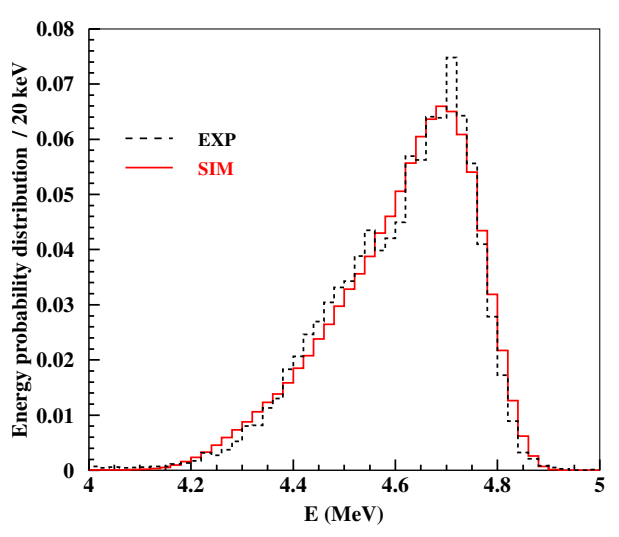

Figure 1. (Color online). Experimental (black dashed line) residual energy spectrum of $\alpha$-particles passing-through a ${ }^{120} \mathrm{Sn}+{ }^{93} \mathrm{Nb}$ foil. The red spectrum is the result of the fit procedure used to extract the target thickness probability distribution. Figure from [6].

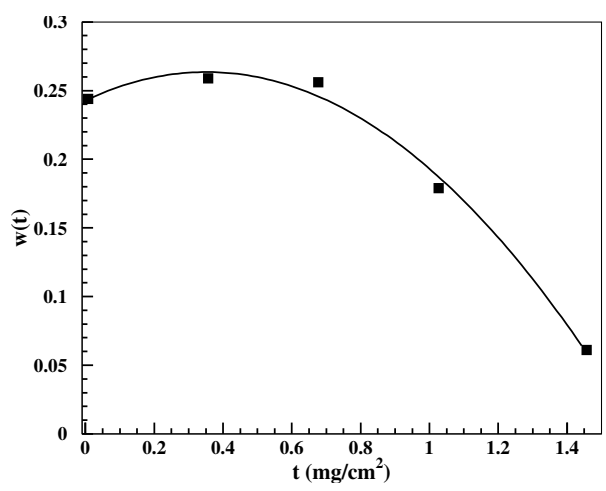

Figure 2. ${ }^{120} \mathrm{Sn}$ target thickness distribution. The continuous line is a polynomial fit of the the $w(t)$. Figure from [6].

prevent their non-uniformity.

\section{An alternative procedure to deduce $\sigma(\mathrm{E})$}

The fitting procedure reported in section 2 is one of the possible ways to solve the problem of finding the best approximation of $\sigma(\mathrm{E})$. An alternative solution to the problem of deducing the excitation function from mean crosssections measured over a broad energy range is suggested in [7]. In [7], an iterative method is used to obtain the fusion excitation function in the case of large beam energyloss in the target. In the experiment reported in [7] the beam energy loss in the target was as large as $40 \mathrm{MeV}$ in the laboratory system. The starting point was plotting the measured cross-section as a function of $\bar{E}$. The obtained curve was fitted with a tensioned spline. The resulting curve was used to calculate $\sigma_{\text {mean }}$ for each measured point $i$ using eq. 8 .

$$
\sigma_{\text {mean }, i}=\int \frac{\sigma(E)}{\frac{d E}{d x}} \frac{d E}{\rho},
$$

where $\frac{d E}{d x}$ is the stopping power of the beam in the target and $\rho$ is the target thickness. Each energy $\mathrm{E}_{i}$, corresponding to the cross section, $\sigma_{\text {mean }, i}$, was obtained by interpolation using the fitting curve. These new points $\left(\sigma_{\text {mean }, i}\right.$, $\mathrm{E}_{i}$ ) were used as inputs for the next iteration of the fit. The procedure was repeated iteratively until convergence was reached. Contrary to what suggested in the present paper, the procedure of [7] does not take account of the correct energy probability distribution of the beam in the target $D_{i}\left(E, t_{0}\right)$, but only of the effect of the energy-loss. Moreover, it is opinion of the present authors that eq. 8, is formally inaccurate; a more formally correct form of eq. 8 reads as:

$$
\sigma_{\text {mean }, i}=\frac{\int \sigma(E) \frac{1}{\frac{d E}{d x}(E)} d E}{\int \frac{1}{\frac{d E}{d x}(E)} d E},
$$

In order to check if the iterative procedure gives the same results as minimising eq. 5, the former is applied to the simulated study of ${ }^{9} \mathrm{Li}+{ }^{120} \mathrm{Sn}$ fusion reaction reported in [6]. The function $\sigma(\mathrm{E})$ is assumed to be given by a Wong-like function [20].

Simulations are performed considering a ${ }^{9} \mathrm{Li}$ beam of about $28 \mathrm{MeV}$ impinging on a stack of 5 foils made of 5 $\mathrm{mg} / \mathrm{cm}^{2}{ }^{120} \mathrm{Sn}$, each followed by $1.5 \mathrm{mg} / \mathrm{cm}^{2}{ }^{93} \mathrm{Nb}$ foil. The correct beam energy probability distribution in the target $D_{i}\left(E, t_{0}\right)$ is calculated in the case of uniform foils and non-uniform foils having Gaussian type non-uniformities of $\mathrm{FWHM}=20 \%$ for ${ }^{120} \mathrm{Sn}$ and $15 \%$ for ${ }^{93} \mathrm{Nb}$. The results are shown in fig. 3 in the case of uniform foils and in fig. 4 for non-uniform foils. Moreover, a comparison is made using the beam energy probability distribution used in [7] i.e. $\frac{1}{\frac{d E}{d x}(E)}$, also shown in figures 3 and 4 .

As can be seen from the figures, the iteration process will give the correct excitation function (red symbols overlap with the full line). However, this is true only if the correct beam energy distributions inside the targets are used. In fact, the points at the lowest energies deviate from the curve in the case $\frac{1}{\frac{d E}{d x}(E)}$ rather than the correct $D_{i}\left(E, t_{0}\right)$ is used, and these deviations are larger for non-uniform foils.

A problem of the iterative approach is, however, the error estimate in the deduced excitation function. In the procedure suggested in the present paper the uncertainties are deduced from the errors in the function parameters. It is not so obvious how to deduce them in the case of the iterative procedure. No mention is made in [7].

\section{The ${ }^{6} \mathrm{Li}_{+}{ }^{120} \mathrm{Sn}$ and ${ }^{7} \mathrm{Li}_{+}{ }^{119} \mathrm{Sn}$ fusion excitation functions}

\subsection{Experiment}

${ }^{6} \mathrm{Li}+{ }^{120} \mathrm{Sn}$ and ${ }^{7} \mathrm{Li}+{ }^{119} \mathrm{Sn}$ fusion cross-sections were measured at INFN-Laboratori Nazionali del Sud (Catania), by using an activation technique based on the off-line detection of the atomic X-rays emitted after the electron capture (EC) decay of the evaporation residues (ER). 


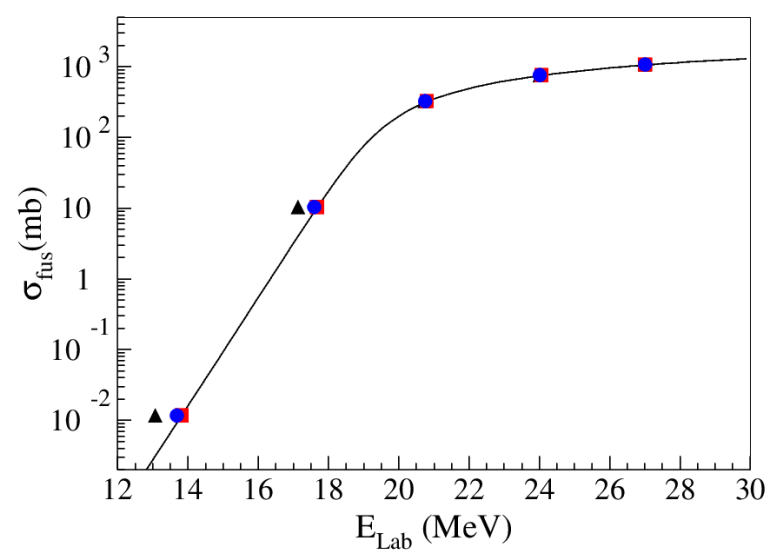

Figure 3. (Color online). Results of the ${ }^{9} \mathrm{Li}+{ }^{120} \mathrm{Sn}$ simulations using the iterative procedure to deduce the fusion excitation function: continuous line, Wong-like function; red squares, excitation function obtained considering the correct $D_{i}\left(E, t_{0}\right)$ in the target for the case of uniform foils; blue circles, excitation function obtained considering only the effect of energy-loss in the target as in [7]. The black triangles correspond to $\sigma_{\text {mean }}$ versus $\bar{E}$.

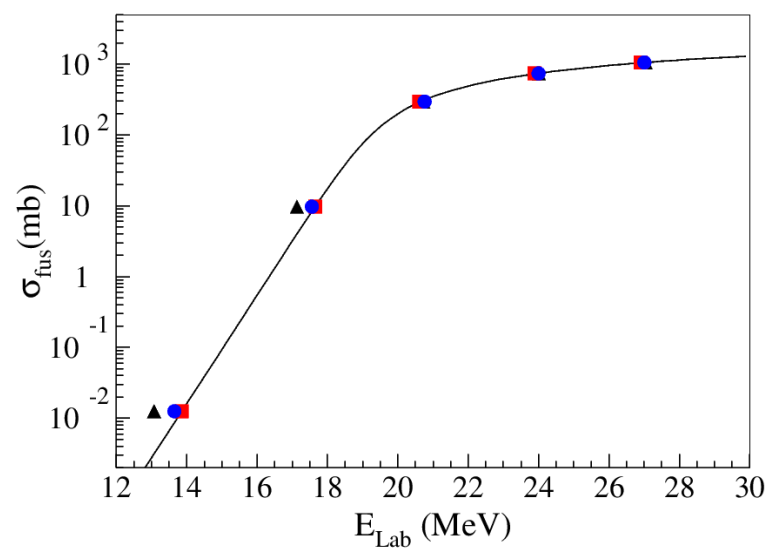

Figure 4. (Color online). Results of the ${ }^{9} \mathrm{Li}+{ }^{120} \mathrm{Sn}$ simulations using the iterative procedure to deduce the fusion excitation function: continuous line, Wong-like function; red squares, excitation function obtained considering the correct $D_{i}\left(E, t_{0}\right)$ in the target for the case of non-uniform foils; blue circles, excitation function obtained considering only the effect of energy-loss in the target as in [7]. The black triangles correspond to $\sigma_{\text {mean }}$ versus $\bar{E}$.

This technique is particularly suitable for these systems since, according to statistical model calculations, the compound nucleus $\left({ }^{126} \mathrm{I}\right)$ decays predominantly by the $3 \mathrm{n}$ and $2 \mathrm{n}$ evaporation channels, producing ${ }^{123} \mathrm{I}\left(\mathrm{t}_{1 / 2}=\right.$ $13 \mathrm{~h}$ ) and ${ }^{124} \mathrm{I}\left(\mathrm{t}_{1 / 2}=4\right.$ days $)$, decaying by EC. Thus, in this case, the complete fusion process can be clearly discriminated against any incomplete fusion or transfer process. The same technique has been used successfully to measure the fusion excitation functions of the systems ${ }^{6,7} \mathrm{Li},{ }^{4,6} \mathrm{He}+{ }^{64} \mathrm{Zn}[1,2,21,22]$.

In the present case, it would have been possible to perform the experiment using single target/catcher foil at each beam energy, due to the high intensity of the
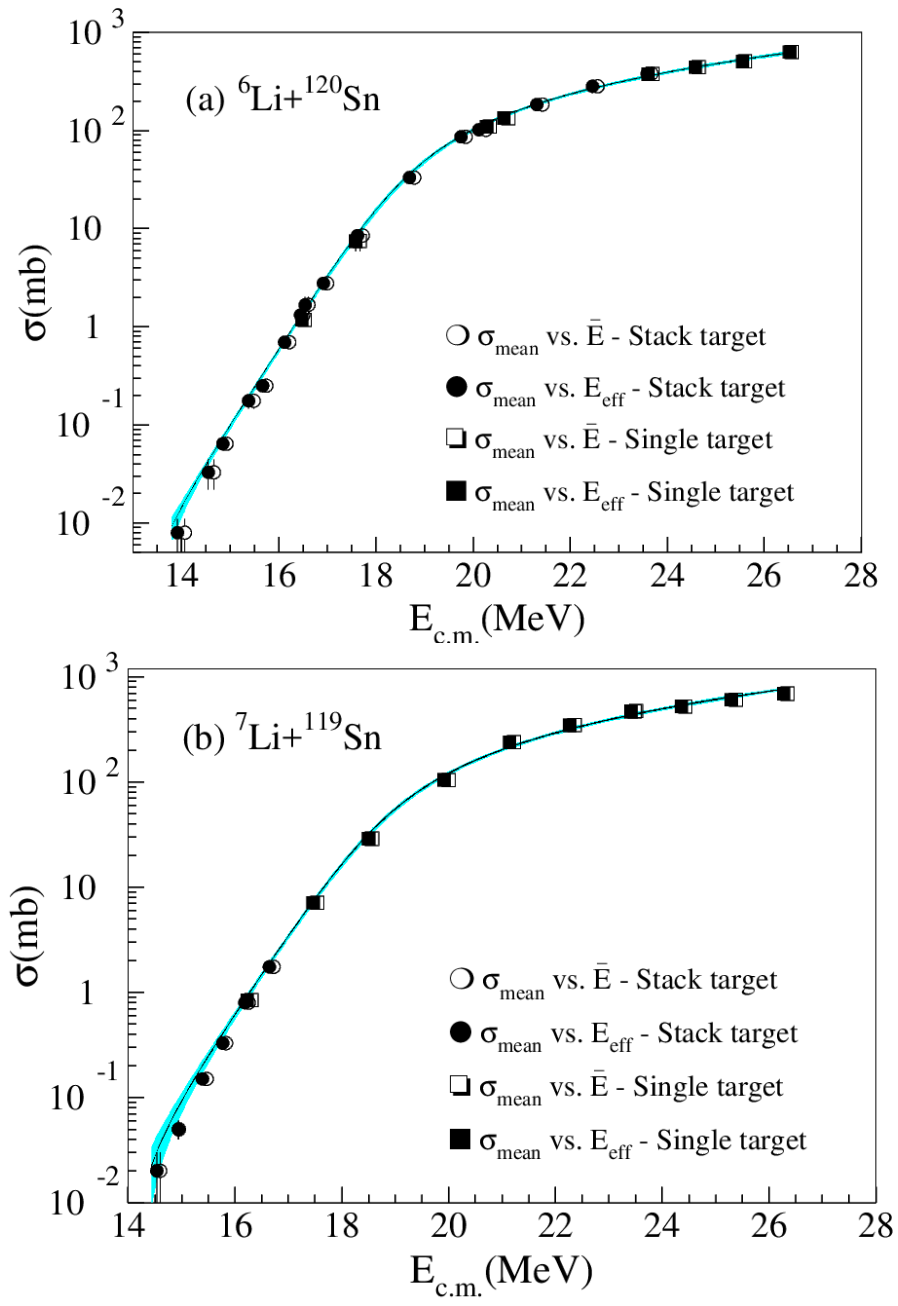

Figure 5. (Color online) Fusion excitation function for a) ${ }^{6} \mathrm{Li}+{ }^{120} \mathrm{Sn}$ and b) ${ }^{7} \mathrm{Li}+{ }^{119} \mathrm{Sn}$ systems extracted as described in detail in [23] (continuous line) and its associated uncertainty (coloured band). The symbols represent the measured crosssections plotted in the usual way i.e. as $\left(\sigma_{\text {mean }}, \bar{E}\right)$ and $\left(\sigma_{\text {mean }}\right.$, $\left.E_{e f f}\right)$. Circles are used for points measured with stacked targets and squares are used for single foil measurements. Figure from [23].

stable beams involved. However, as a test for a future experimental program with unstable lithium isotopes, the stacked target technique is used instead. The unfolding procedure is therefore required to deduce the complete fusion excitation function. Details of the experiment are reported in [23].

For each irradiated foil the thickness probability distribution function $w(t)$ is extracted. Known $w(t)$ it is possible to calculate, using SRIM, the beam energy probability distribution $D_{i}\left(E, t_{0}\right)$ for each measured point of the excitation function. Using the unfolding procedure described in section 2 the fusion excitation function for the two reactions is extracted, and it is shown in fig. 5. An important check to be done in order to verify that the functional form obtained using the unfolding procedure is a good representation of the fusion excitation function, is to plot the ratio between the $\sigma_{\text {calc-mean, } i}$, calculated from 

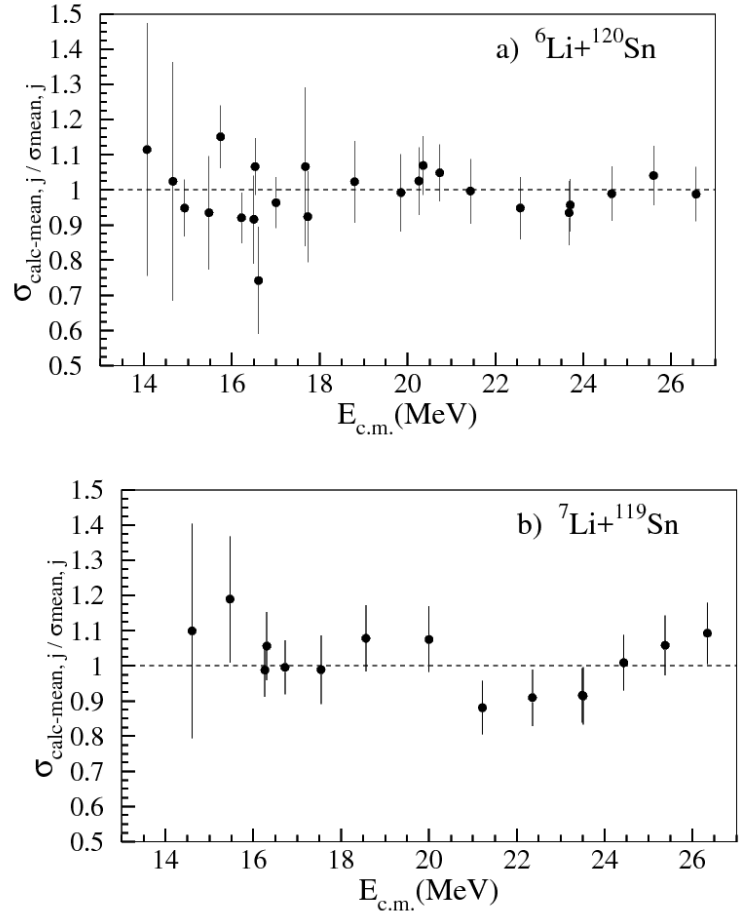

Figure 6. Ratio between $\sigma_{\text {calc-mean }, \text { and }} \sigma_{\text {mean }, i}$ for the reactions: ${ }^{6} \mathrm{Li}+{ }^{120} \mathrm{Sn}$ a), and ${ }^{7} \mathrm{Li}+{ }^{119} \mathrm{Sn}$ b). Figure from [23]

the functional form $\hat{g}(E, \mu)$ averaged over the beam energy distribution in the target $D_{i}\left(E, t_{0}\right)$, and the measured one $\sigma_{\text {mean }, i}$. If this ratio is $\approx 1$ in the whole energy range then the procedure is self consistent, and the extracted functional form is a good representation of the fusion excitation function. This was verified to be the case of the present analysis, and the ratio for the two reactions is shown in fig. 6.

\subsection{Results}

At energies above the Coulomb barrier the complete fusion cross-section, in the case of reactions induced by weakly bound beams, shows a suppression with respect to the 1D-Barrier Penetration Model (BPM) and/or the Coupled Channel (CC) calculations which do not take coupling to continuum or transfer into account ([16] and reference therein). It is found that the suppression factor depends on the breakup threshold of the projectile e.g. [16].

In order to deduce the suppression factors of the complete fusion cross-section for the two systems ${ }^{6} \mathrm{Li}+{ }^{120} \mathrm{Sn}$ and ${ }^{7} \mathrm{Li}+{ }^{119} \mathrm{Sn}$, the cross-sections were reduced according to the reduction procedure introduced by Canto et al. $[14,15]$. Once the data are reduced, the complete fusion suppression factor is extracted by comparing them with a benchmark function, called Universal Fusion Function (UFF) defined in [14, 15]. In fig. 7 such a comparison is shown. In fig. 7 a, the reduced cross-sections are shown in logarithmic scale and in fig. $7 \mathrm{~b}$ in linear. The
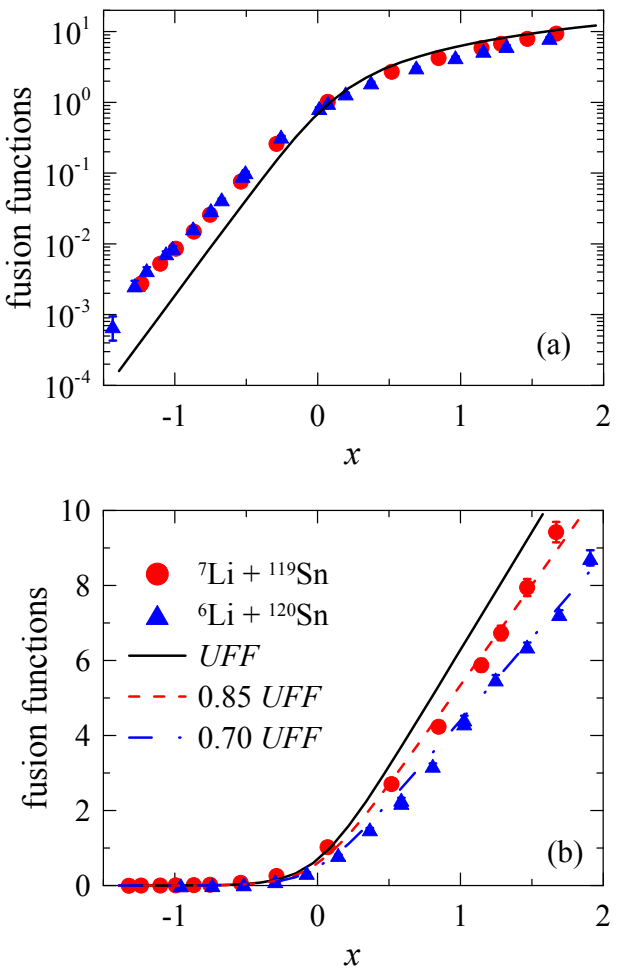

Figure 7. (Color online) Comparison of $\mathrm{CF}$ reduced cross section for the ${ }^{6} \mathrm{Li}+{ }^{120} \mathrm{Sn}$ and ${ }^{7} \mathrm{Li}+{ }^{119} \mathrm{Sn}$ systems with the UFF. The experimental data reported in the figure correspond to $\left(\sigma_{\text {mean }, i}\right.$, $\left.E_{d e c, i}\right)$, where $E_{d e c, i}$ is the energy point of deconvolution curve which corresponds to the measured cross-section $\sigma_{\text {mean }, i}$. Figure from [23].

Coulomb barrier corresponds to $x=0$. From fig. 7, it can be seen that by comparing the experimental reduced fusion cross-section with the UFF, for both reactions, the complete fusion is enhanced at energies below the barrier and hindered above. The deduced suppression factor above the barrier for the ${ }^{6} \mathrm{Li}+{ }^{120} \mathrm{Sn}$ system is $0.70 \pm 0.05$, and for the ${ }^{7} \mathrm{Li}+{ }^{119} \mathrm{Sn}$ system is $0.85 \pm 0.05$. Such values, while confirming the breakup threshold dependence of the suppression factors, appear to be slightly nearer to unity than the ones found in the systematic of Wang et al. [17] for other systems.

At energies below the barrier, as it was already observed in collisions of ${ }^{6,7} \mathrm{Li}$ with different targets e.g. [16], the UFF underestimates the data. Since coupling to continuum and transfer channels are not taken into account in the reduction of the data, they are responsible for the underestimation of the UFF.

What is interesting to note, is that below the barrier the two sets of reduced data (fig. 7 a) are very close in spite of the different $\mathrm{n}$-transfer $\mathrm{Q}_{\text {value }}$. This behavior seems to be consistent with the one observed in fusion reactions of heavier systems [24-26] and appears to confirm the idea, proposed by [27-29], that the mechanism that produces the enhancement of the sub-barrier fusion cross-section may be rather complex, and that the relevant effects is not limited to the difference in the $\mathrm{n}$-transfer $\mathrm{Q}_{\text {value }}$. CRC calculations were also performed for the ${ }^{7} \mathrm{Li}+{ }^{119} \mathrm{Sn}$ which 
has the largest positive $\mathrm{Q}_{\text {value }}$ for $\mathrm{n}$-transfer (details can be found [23]), and they confirm that the effect of the coupling with $\mathrm{n}$-transfer channel produces negligible effects.

\section{Summary and conclusions}

This paper highlights the problem associated with the extraction of the correct excitation function in the case where cross-sections strongly varying with energy, with beams having large energy dispersion, are measured. These experiments are typically the sub-barrier fusion excitation function measurements with radioactive beams, where it is necessary to maximise the experimental yields due to the low intensity of the beams combined with the small cross-sections involved.

It has been demonstrated that in the case of large beam energy dispersion, there are ambiguities in associating the effective beam energy to the measured mean cross-section and this can lead to an error in the determination of the excitation function. In fact, plotting $\sigma_{\text {mean }}$ either versus $\mathrm{E}_{\text {mean }}$ or $\mathrm{E}_{\text {eff }}$ will not give the correct excitation function, and then a significant misinterpretations of the data may arise. Accurate information can be obtained from the experimentally measured $\sigma_{\text {mean }}$, provided that a full characterisation of the targets is done. In such a way, it is possible to calculate for every target the beam energy probability distribution $D\left(E, t_{0}\right)$ function, which specifies how the cross-section will be integrated over the beam energy.

This deconvolution procedure is used to deduce complete fusion excitation functions for the two reactions, ${ }^{6} \mathrm{Li}+{ }^{120} \mathrm{Sn}$ and ${ }^{7} \mathrm{Li}+{ }^{119} \mathrm{Sn}$ in the c.m. energy range from 14 to $28 \mathrm{MeV}$. The measured cross-sections, renormalised according to $[14,15]$, were compared to the UFF. The experimental cross sections are found to be suppressed with respect to the UFF at energies above the Coulomb barrier. The suppression factor for ${ }^{6} \mathrm{Li}+{ }^{120} \mathrm{Sn}$ is found $0.7 \pm 0.05$, and for ${ }^{7} \mathrm{Li}+{ }^{119} \mathrm{Sn}$ is found $0.85 \pm 0.05$. These factors are a little smaller than the ones obtained with heavier targets.

Below the Coulomb barrier, the renormalized complete fusion cross-sections are very similar even though the $\mathrm{Q}_{\text {value }}$ for neutron transfer are substantially different. Therefore, the mechanism that produce the sub-barrier enhancement, experimentally observed in some reactions, must be more complicated than just the difference in the $\mathrm{Q}_{\text {value }}$.

\section{References}

[1] A. Di Pietro et al. Phys. Rev. C 69, 044613 (2004)

[2] V. Scuderi et al. et al. Phys. Rev. C 84, 064604 (2011)

[3] J. J. Kolata et al. Phys. Rev. Lett. 81, 4580 (1998)

[4] C. Signorini et al. Nucl. Phys. A 735, 329 (2004)

[5] R. Wolski et al. Eur. Phys. Jour. A 47, 111 (2011)

[6] M. Fisichella et al. Phys. Rev. C 92, 064611 (2015)

[7] J. F. Liang et al. Phys. Rev. C 75, 054607 (2007)

[8] T. H. Stelson et al. Phys. Rev. C 41, 1584 (1990)

[9] R. A. Broglia et al. Phys. Rev. C 27, 2443 (1983)

[10] S. Kalkal et al. Phys. Rev. C 81, 044610 (1990)

[11] H. Q. Zhang et al. Phys. Rev. C 82, 054609 (2010)

[12] A. M. Stefanini et al. Phys. Rev. C 52, R1727 (1995)

[13] M. Dasgupta et al. Phys. Rev. C 70, 024606 (2004)

[14] L. F. Canto et al. Jour. Phys. G 36, 015109 (2009)

[15] L. F. Canto et al. Nucl. Phys. A 821, 51 (2009)

[16] L. F. Canto et al. Phys. Rep. 596, 1 (2015)

[17] B. Wang et al. Phys. Rev. C 90, 034612 (2014)

[18] BJ. F. Ziegler et al. Nucl. Inst. Meth. B 268, 1818 (2010)

[19] J. F. Ziegler, SRIM code (http://www.srim.org)

[20] C. Y. Wong Phys. Rev. Lett. 31, 766 (1973)

[21] A. Di Pietro et al. Eur. Phys. Jour. S. T. 150, 15 (2007)

[22] A. Di Pietro et al. Phys. Rev. C 87, 064614 (2013)

[23] M. Fisichella et al. Phys. Rev. C 95, 034617 (2017)

[24] Z. Kohley et al. Phys. Rev. Lett. 107, 202701 (2011)

[25] H. M. Jia et al. Phys. Rev. C 86, 044621 (2012)

[26] A. M. Stefanini et al. Eur. Phys. Jour. A 49, 1 (2013)

[27] V. V. Sargsyan et al. Phys. Rev. C 85, 024616 (2012)

[28] V. V. Sargsyan et al. Phys. Rev. C 91, 014613 (2015)

[29] V. A. Rachkov et al. Phys. Rev. C 90, 014614 (2014) 